\title{
Paradigmas en Odontología
}

\section{Paradigms in Dentistry}

\author{
José Francisco Gómez Clavel*
}

En diferentes ámbitos de la cultura, la educación y las ciencias se plantea el cambio de paradigma, cambiar las ideas preconcebidas (paradigm shift) para tener mejores resultados.

Un paradigma se conforma de un cuerpo de conocimientos teóricos, un conjunto de preceptos metodológicos y el modo de generar conocimientos que una comunidad académica ha adoptado como guía de su quehacer cotidiano; ya sea para dirigir sus discusiones o el camino de sus investigaciones y la interpretación de los datos que arrojan sus experimentos $u$ observaciones. ${ }^{1}$

También un paradigma guía en las profesiones el cómo, quién y por qué se realizan las actividades prácticas, cómo abordan sus problemas o los problemas que la sociedad les plantea a resolver. En nuestro caso, la identificación y valoración de lesiones, o bien, el diagnóstico y el tratamiento de las afecciones de la cavidad oral.

¿Cuál es nuestro paradigma en el caso de la caries? ¿Seguimos viendo a la caries como un proceso irreversible, como lesiones cavitadas?

Ya en 2004 Fejerskov nos plantea revisar el concepto que tenemos del mecanismo de acción de los fluoruros en la prevención y el control de la caries, y si ésta es una enfermedad infecciosa en el sentido clásico de este concepto. ${ }^{2}$

En la conferencia «Diagnóstico y manejo de la caries dental a lo largo de la vida», el Instituto Nacional de Salud de los Estados Unidos de América (2001), reconoció un cambio de paradigma en el manejo de la caries como un proceso de enfermedad. El cambio del modelo quirúrgico que se centra en la restauración de las estructuras del diente a un modelo que se enfoque en la prevención y el control de la caries a través del manejo de los ciclos de desmineralización y remineralización de las estructuras dentales con el objetivo de preservar los dientes para toda la vida. ${ }^{3}$

Con el objetivo de manejar de forma más eficiente las medidas preventivas dirigidas de manera particular a cada paciente, se ha propuesto el manejo de la caries a través de la valoración del riesgo, para ade- cuar en cada paciente las medidas necesarias para un adecuado control de la caries, a través de una serie de técnicas que se conocen como odontología de mínima intervención, que pone el énfasis en el diagnóstico y tratamiento de las lesiones no cavitadas. ${ }^{4}$

¿Lesiones no cavitadas de caries? Sí, el diagnóstico de éstas puede sistematizarse a través de ICDAS (International Caries Detection and Assessment System). Este sistema mide cualitativamente las diferentes etapas del proceso de caries desde el esmalte sano (código 0) hasta las lesiones cavitadas con pérdida considerable de la estructura dental (código 6). ${ }^{5}$

En la educación, los docentes creemos y apostamos por la forma en la que conceptualizamos nuestra docencia; tradicionalmente nos visualizamos como los poseedores del saber, nombramos a los jóvenes que formamos, alumnos a los que hay que alimentar e iluminar con nuestra sabiduría, y como una de las funciones de la universidad (el templo del saber) es la docencia, pues la función del docente es la de convertirse en faro de luz para iluminar a los alumnos. Nuestras prácticas docentes se centran en la conferencia o la clase magistral. Además, nuestra experiencia y la forma en la que fuimos formados avalan esta metodología didáctica. Sin embargo, en 1998 la UNESCO en su Declaración Mundial sobre la Educación Superior en el Siglo XXI, ${ }^{6}$ nos llama a desarrollar un nuevo modelo de enseñanza superior, que debe estar centrado en el estudiante.

¿Qué implicaciones tiene este nuevo modelo? Que el estudiante sea capaz de buscar, analizar y utilizar la información que se genere por la investigación, y pueda transferirla a la práctica clínica después de realizar la valoración de las evidencias científicas que

\footnotetext{
* Carrera de Cirujano Dentista. Laboratorio de Investigación en Educación y Odontología. Facultad de Estudios Superiores Iztacala, UNAM.
}

Este artículo puede ser consultado en versión completa en http://www.medigraphic.com/facultadodontologiaunam 
respaldan a la información de nuevos tratamientos o al escrutinio de las prácticas prevalentes, que desarrolle el pensamiento crítico a través de estrategias en las que el estudiante realice un aprendizaje activo en escenarios educativos en donde aprendan a través de cuestionamientos y retos como en la estrategia didáctica de aprendizaje basado en problemas (ABP). ${ }^{7}$

El tercer cambio al que somos convocados está relacionado con la forma en la que hemos ejercido nuestra práctica clínica, una práctica en solitario, la formación profesional del odontólogo está orientada de manera importante al tratamiento clínico individual. Sin embargo, las características de la población que ahora atendemos, pacientes con polifarmacia, como el diabético, el postinfartado o con otras patologías hacen que ahora tengamos la necesidad de trabajar en equipos interprofesionales, por lo que debemos introducir a nuestros estudiantes en esta práctica a través de experiencias educativas que ya son promovidas y evaluadas por el organismo acreditador en la Unión Americana. ${ }^{8}$ Las universidades de Norteamérica tienen que introducir cursos que promuevan que los estudiantes de odontología interactúen con estudiantes de otras profesiones para aprender sobre, desde y entre sí, permitiendo la colaboración efectiva para mejorar los resultados de salud, ${ }^{9}$ ya que la prevención y el tratamiento de las enfermedades puede realizarse mejor cuando los miembros del equipo de trabajo de atención bucodental trabajan juntos en colaboración, así como con miembros de otras profesiones de la salud.

El gran reto que tenemos los educadores en la odontología es adecuarnos, con una visión crítica, fundamentada, que sea producto del análisis de las evidencias que se generan en nuestro campo teniendo en cuenta que al incorporarnos a estos nuevos paradigmas, no lo hagamos de manera irreflexiva, no seamos forzosamente los primeros, pero tampoco seamos los últimos.

\section{REFERENCIAS}

1. Hernández-Rojas G. Paradigmas en psicología de la educación. México: Paidós Educador; 1998.

2. Fejerskov $\mathrm{O}$. Changing paradigms in concepts on dental caries: consequences for oral health care. Caries Res. 2004; 38 (3): 182-191.

3. Diagnosis and management of dental caries throughout life. National Institutes of Health Consensus Development Conference statement, March 26-28, 2001. J Dent Educ. 2001; 65: 11621168.

4. Frencken JE, Peters MC, Manton DJ, Leal SC, Gordan VV, Eden E. Minimal intervention dentistry for managing dental caries-a review: report of a FDI task group. Int Dent J. 2012; 62 (5): 223243.

5. Ismail Al, Sohn W, Tellez M, Amaya A, Sen A, Hasson $\mathrm{H}$ et al. The International Caries Detection and Assessment System (ICDAS): an integrated system for measuring dental caries. Community Dent Oral Epidemiol. 2007; 35 (3): 170-178.

6. UNESCO. Conferencia Mundial sobre la Educación Superior. La educación superior en el siglo XXI. Visión y acción. Artículo 9. Métodos educativos innovadores: pensamiento crítico y creatividad. 1998.

7. Fincham AG, Shuler CF. The changing face of dental education: the impact of PBL. J Dent Educ. 2001; 65 (5): 406-421.

8. Commission on Dental Accreditation. Accreditation standards for dental education programs. Chicago: American Dental Association. Available in: http://www.ada.org/ /media/CODA/Files/ predoc_2013.ashx

9. World Health Organization (WHO). Framework for action on interprofessional education \& collaborative practice. Geneva: World Health Organization; 2010. Available in: http://whqlibdoc. who.int/hq/2010/WHO_HRH_HPN_10.3_eng.pdf

Dirección para correspondencia: José Francisco Gómez Clavel E-mail: gomclave@gmail.com 